\title{
Kepler-K2: A Search for Very Red Variables
}

\author{
Erich Hartig ${ }^{1, \star}$, Kenneth Hinkle ${ }^{2, \star \star}$, and Thomas Lebzelter ${ }^{1, \star \star \star}$ \\ ${ }^{1}$ University of Vienna, Department of Astrophysics \\ ${ }^{2}$ National Optical Astronomy Observatories, P.O. Box 26732, Tucson, AZ 85726 USA
}

\begin{abstract}
As a monitoring mission the NASA Kepler K2 mission offers the opportunity to search for long period variables, in this case along the ecliptic plane. The usability of $\mathrm{K} 2$ for this search is limited since the observing period of an individual target is restricted to about 80 days. However, this time scale allows detecting LPVs and making at least a rough estimate of the period length. Since no ready-to-use light curves were provided during the early phases of the $\mathrm{K} 2$ mission, we had to use a reduction algorithm developed by our group based on the experiences from the primary Kepler mission. Our results can now be nicely compared with the K2 'High Level Science Products' released in June 2016. The first results of our study up to campaign 3 are presented.
\end{abstract}

\section{Introduction}

Long period variables (LPVs) are highly evolved stars of low and intermediate mass. They are characterized by large amplitude variations in the visual, periods of a few 10 to several 100 days and very red colour due to both the low surface temperature and the circumstellar reddening by dust. As a consequence we chose stars with 2MASS colours J-Ks greater than 0.8 and $\mathrm{Ks}<8.0$ mag as targets. An additional selection criterion was the availability of basic information from Simbad, GSC 2.3, 2MASS, and AAVSO data bases to ensure we select stellar objects only. Figure 1 shows the interesting objects in the colourmagnitude diagram of campaign 1 . In particular, we were interested in detecting highly reddened long period variables (LPVs), which is a main group of stars found with these selection criteria. To focus on these objects we set a lower period limit of 10 days. Most of the stars with very large amplitudes, usually dubbed 'Miras', have been detected as variables before, so the main interest goes into stars with small to moderate light amplitudes.

\section{Data Preparation}

Even more than for the Kepler primary mission (see Hartig et al. [4]) the data reduction had to be done with care to extract the true stellar signal. Dealing with LPVs we had to expect a variability on a time scale comparable to the length of the data set. Therefore, different to studies of other types of variables, it was a particular challenge to distinguish instrumental trends from long time variability.

\footnotetext{
^e-mail: erich.hartig@univie.ac.at

$\star \star$ e-mail: khinkle@noao.edu

$\star \star \star$ e-mail: thomas.lebzelter@univie.ac.at
}

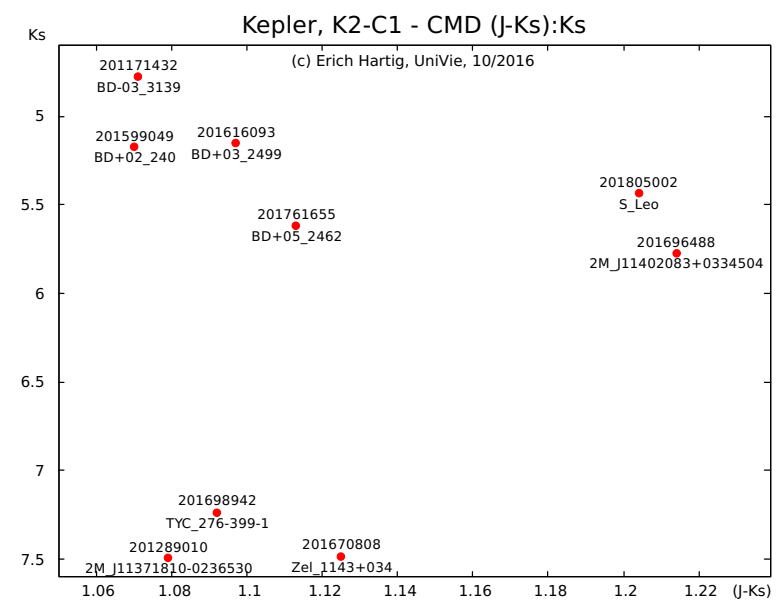

Figure 1: K2 - C1, Colour Magnitude Diagram (CMD) (J-Ks):J.

For the data preparation we adjusted our pipeline software for TPD, which we had used for data from the $\mathrm{Ke}$ pler primary mission, and modified it to the $\mathrm{K} 2$ requirements. While the 6 hours signal caused by the firing of the thrusters for correcting the telescope's pointing could be easily corrected, a non-linear flux increase over each observation period had to be removed up to campaign $\mathrm{C} 2$. Furthermore, it turned out that stars with magnitudes $<8.5$ Kepmag typically show elongated point spread functions. To circumvent this problem we applied a non-standard pixel aperture for these objects, which allowed to improve the light curve's signal-to-noise ratio significantly. Some examples are shown in Figures 4 to 10. 


\section{Background Trend}

We processed 18 targets of the target pixel files (TPF) from the 8 days two-wheel engineering test (K2-CEng) and observed an increase of the flux of a number of targets over time, which follows approximately the background increase, see Figure 2. We calculated the background from pixels around the mask and tested two strategies to remove this trend from the target pixel data (TPD):

- by using the median of the background

- by using the average of 6 stars with small amplitude variations showing the long-time trend (see Figure 2, EPIC 60020773).

Once released we compared our results with the ' $\mathrm{K} 2$ High Level Science Product' (HLSP) K2SFF.

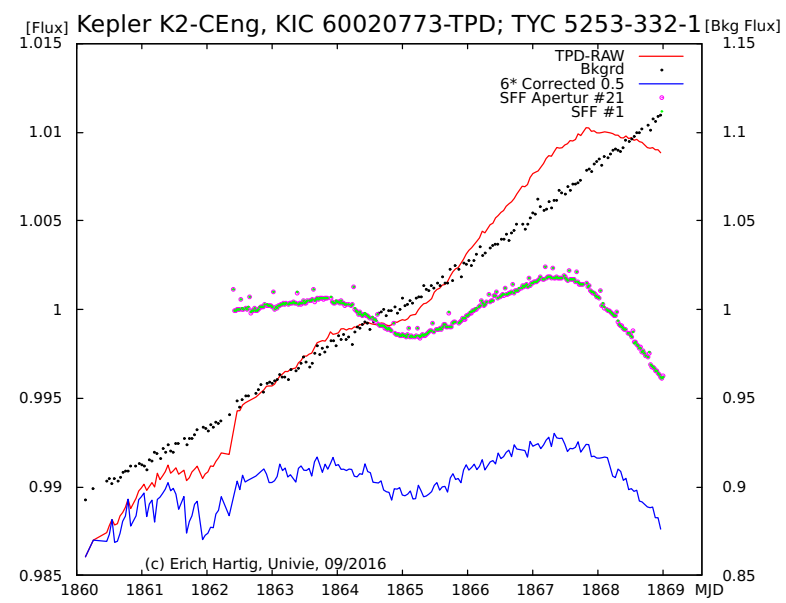

Figure 2: K2 - Engineering Test Data, EPIC 60020773, relative flux of raw TPD (red) compare with adjusted TPD (blue), K2SFF aperture 1 (green), aperture 21 (purple) and background (black).

\section{K2 Campaigns}

\subsection{K2 Campaign 0 (K2 - C0)}

The K2-C0 campaign was a full length engineering test for the $\mathrm{K} 2$ concept but the spacecraft was not in fine-point mode for the first part of $\mathrm{C} 0$ and we treated these parts with care. Only 9 of our 62 requested targets were observed.

We find a number of stars, which, similar to the engineering test, show an increase of brightness by about $0.5 \mathrm{Kepmag}$, approximately following the increase of the background. In Figure 3 the TPD light curve retrieved for C-star EPIC 202070319, the corrected TPD light curve using the average of six reference stars, and the K2SFF results of aperture 1 and 21 are compared with the background.

Unfortunately, we found no unique relation of the adjustment factor for these long-time variations for all candidates, K2SFF again provides an acceptable solution. The exception is EPIC 202062457, SU Gem. K2SFF skips the first part of the data because Kepler achieved fine pointing control only for the last approximately 35
Table 1: Number of Candidates

\begin{tabular}{lll}
\hline Campaign & Requested & Observed \\
\hline C0 & 62 & 9 \\
C1 & 68 & 34 \\
C2 & 48 & 41 \\
C3 & 35 & 12 \\
\hline
\end{tabular}

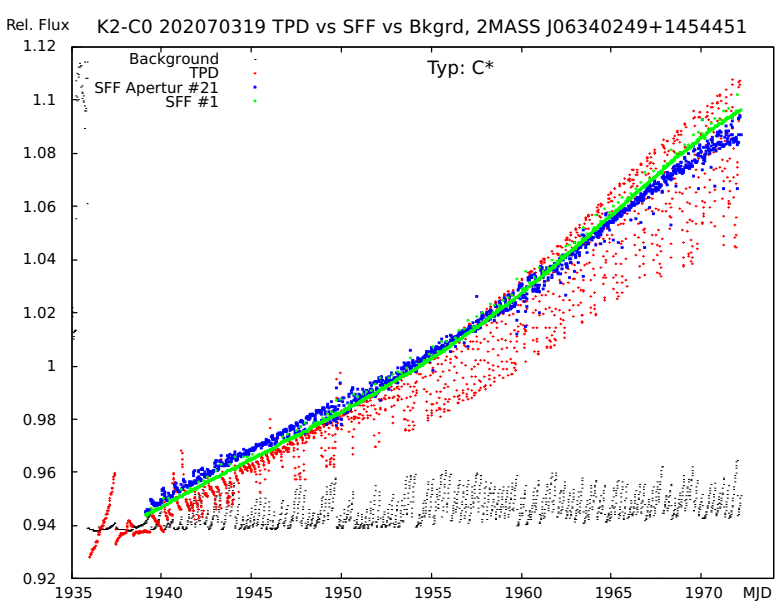

Figure 3: K2-C0, C-star EPIC 202070319, relative flux of background (black) compared with TPD (red), K2SFF aperture 1 and 21 (blue/green).

days; with the inclusion of the first part of the TPD data with our software we could improve the results. The TPD calculation gave a period of $25.158 \mathrm{~d}$, which fits better to the AAVSO result of $50.0 \mathrm{~d}$ (half the period) than the K2FF period of 27.213d. We used the software package Period04 by Lenz P. and Breger M., [5] for their calculations.

In Figure 4 we show five light curves of our candidates, which indicates long periods.
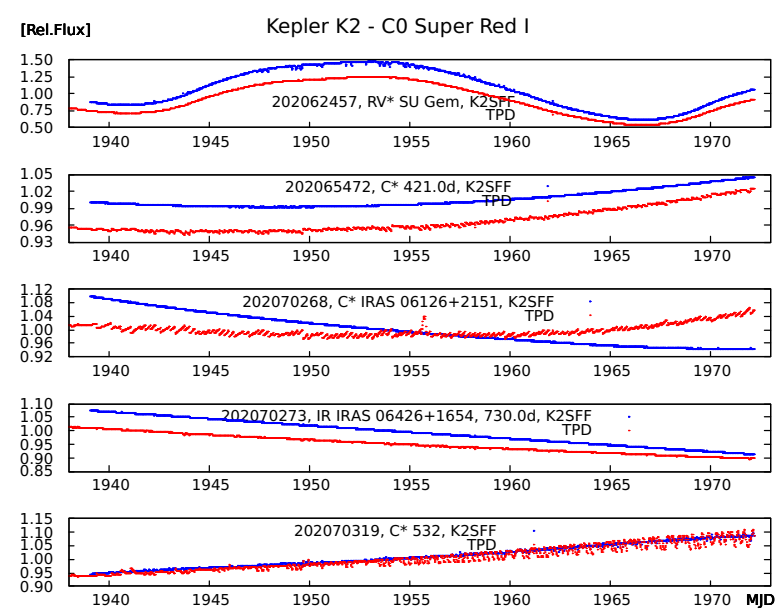

Figure 4: K2 - C0 LPVs, TPD (red), K2SFF aperture 21 (blue). 


\subsection{K2 Campaign 1 (K2 - C1)}

We applied the $\mathrm{C} 0$ correction also to the $\mathrm{K} 2-\mathrm{C} 1$ data to remove the long-time trend by using the average of six similar stars improving the determination of the amplitude and the long period. Only 3 of our requested objects are known LPVs, one Mira and two C-stars, showing periods longer than $10 \mathrm{~d}$. We additionally scanned the observed objects by ( $\mathrm{J}-\mathrm{Ks}$ ) colour and $\mathrm{Ks}<8.0$ and found 12 more LPV candidates, the top five are shown in Figure 5. The last one, EPIC 201368935, TT Leo, does not show a significant variation in the SFF data.

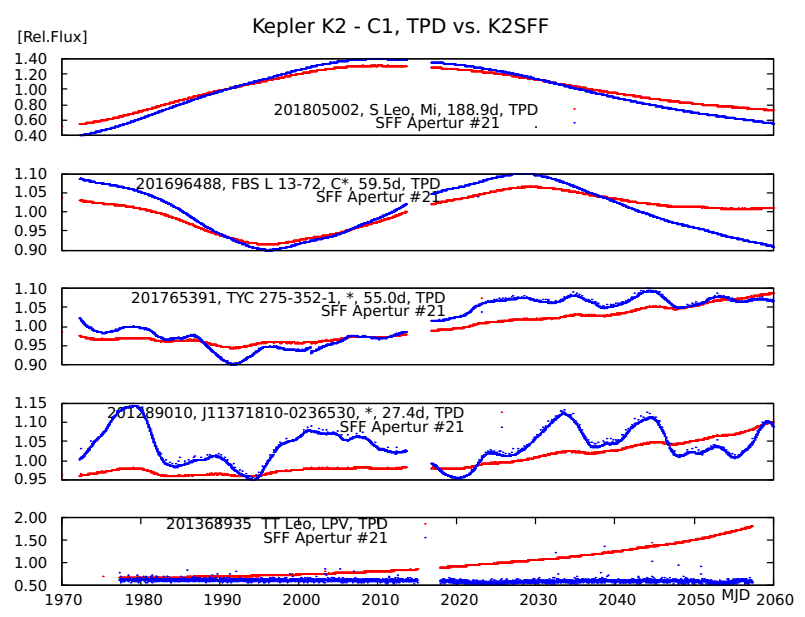

Figure 5: K2 - C1, Top 5 LPVs, TPD (red), K2SFF aperture 21 (blue).

\subsection{K2 Campaign 2 (K2 - C2)}

In K2-C2 the long-time disturbance still exists and has been corrected as described before. Some light curves derived with our own TPD analyses compared nicely with K2SFF, see Figure 6 and 7, some do not. From our 48 requested targets 41 have been observed. We found 21 possible candidates showing long periods, 5 of them are Miras, the rest are classified as infrared sources. Only V1158 Sco (EPIC 205240599) is a presently known LPV.

\subsection{K2 Campaign 3 (K2 - C3) - K2SFF A new hope?}

Finally, K2-C3 provided simple aperture photometry (SAP_FLUX) and a more processed version of SAP, with artifact mitigation included, called pre-search data conditioning flux (PDCSAP_FLUX) light curves (Smith, J. C. et al., [6], Stumpe, M. C. et al., [7]) for long cadence data. Three corrected light curves are generated: (a) a Bayesian MAP fit, (b) a Robust Least Squares fit and (c) no correction. PDC then selects the fit that results in the best photometric precision ${ }^{1}$.

Background pixels were observed and used to model the background level across the field of view. The calibrated pixels available in the target pixel files now have this background level removed.

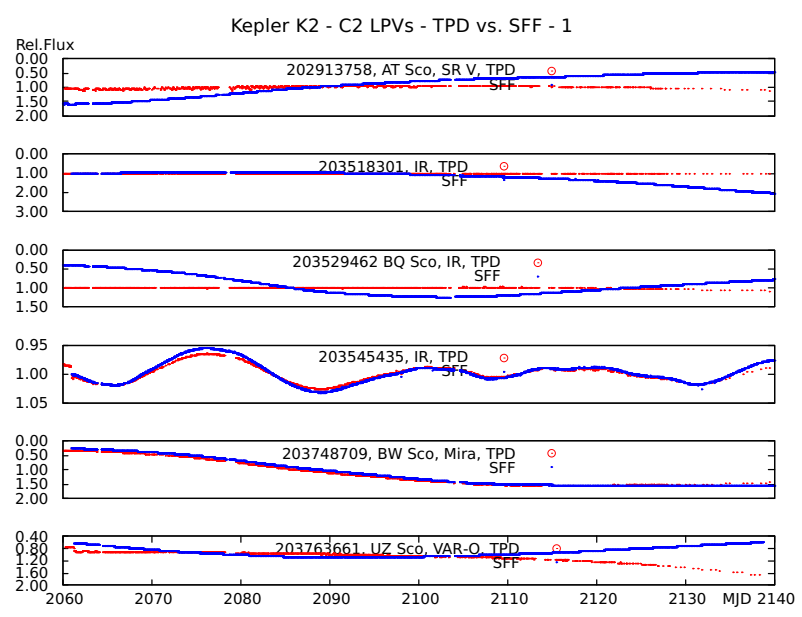

Figure 6: K2 - C2 sample 1 of LPVs, TPD (red), K2SFF aperture 21 (blue).

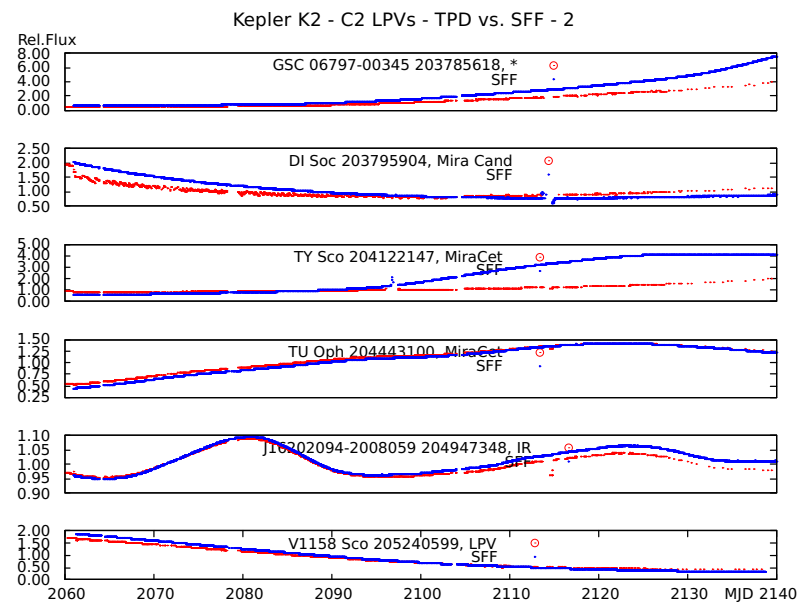

Figure 7: K2 - C2 sample 2 of LPVs, TPD (red), K2SFF aperture 21 (blue).

The new "High Level Science Products"2 had been additionally tested and we found that K2SFF reproduces LPVs usually best, K2-everest did not provide data for this target yet. K2SFF is a technique for extracting and correcting K2 photometry similar to those developed for Spitzer but optimized to the peculiarities of the K2 mission, see Vanderburg A., and Johnson J. A. [8] and Vanderburg A [9]. In few cases we observe an amplitude jump at MJD 2189.052, see Figure 8, EPIC 206427151. The long-time trend of increasing brightness is no longer visible here and K2SFF preserved the long periods too. From our 35 requested objects 12 have been observed. In Figure 9 and 10 we show the 12 light curves and compare our TPD results with K2FF.

\section{Conclusions}

We noticed that stars with magnitudes $<8.5$ Kepmag typically show elongated pixel configurations.

\footnotetext{
${ }^{1}$ https://keplerscience.arc.nasa.gov/K2/C3drn.shtml

${ }^{2}$ http://archive.stsci.edu/archive_news/2016/06-Jun/index.html
} 


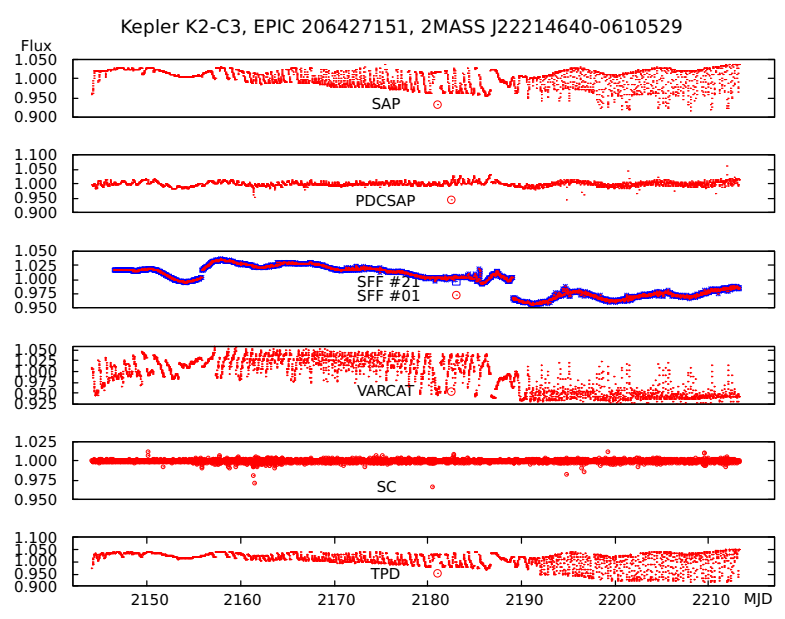

Figure 8: Kepler EPIC 206427151 Compare LCs with High Level Science Products like K2SFF, K2VARCAT, and K2SC.

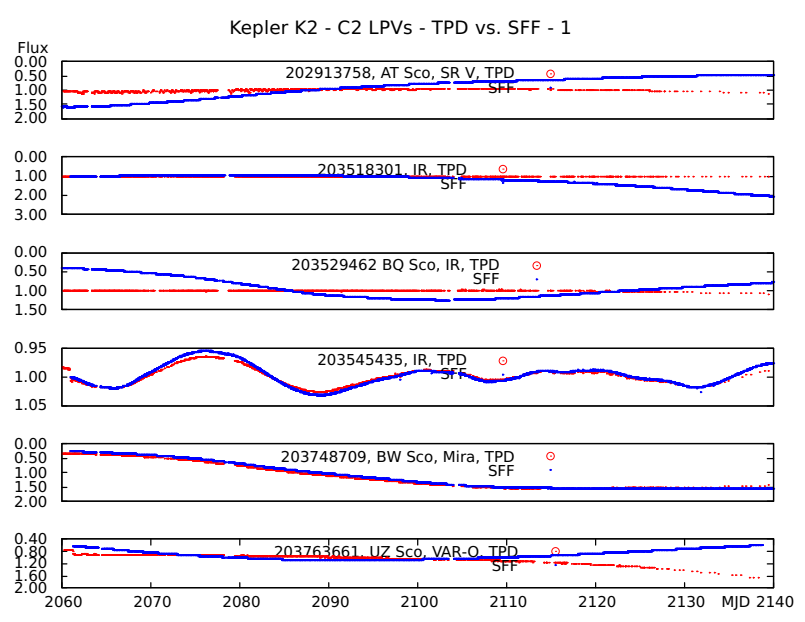

Figure 9: Kepler EPIC K2 - C3 sample 1 of LPV candidates.

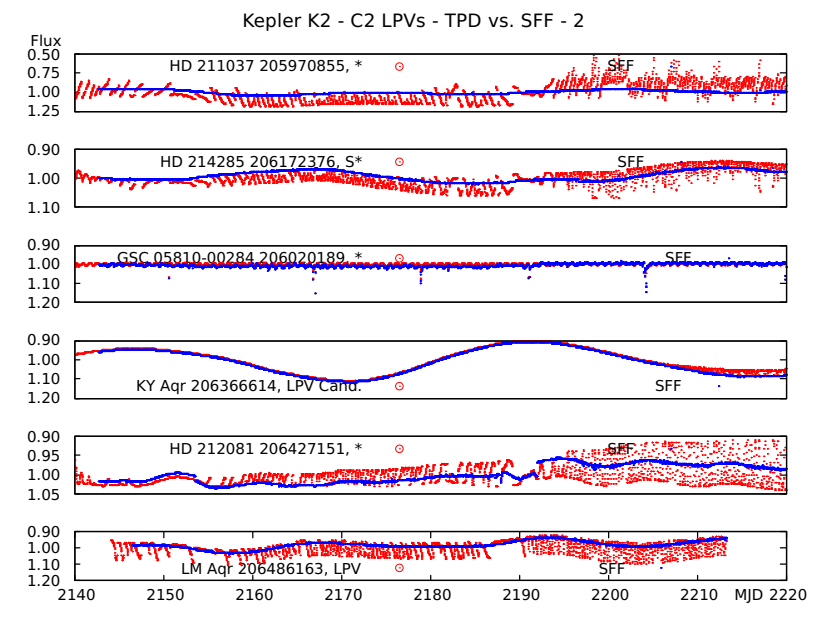

Figure 10: Kepler EPIC K2 - C3 sample 2 of LPV candidates.
Our software allows the specific selection of pixel masks, which gives good results but fails in the sample above. We will therefore continue all further analysis additionally to our own software package with K2SFF.

\section{Acknowledgements}

EH thanks the University of Vienna for support (FA538905). TL was funded by the FWF project P23737.

\section{References}

[1] Aigrain, S. et al., 2016, MNRAS, 459, 2408

[2] Armstrong, D. J., Osborn, H. P., Brown, D. J. A., et al., 2014, e-prints arXiv:1411.6830

[3] Armstrong, D. J., Kirk, J., Lam, K. W., et al., 2016, MNRAS,456, 2260

[4] Hartig, E., Cash, J., Hinkle, K., Lebzelter, T., Mighell, K., and Walter, D., 2014, AJ, 148, 123

[5] Lenz, P., Breger, M. 2005, Communications in Astrosysmologie, 146, 53

[6] Smith, J. C., Stumpe, M. C., Van Cleve, J. E., et al., 2012, arXiv:1203.1382

[7] Stumpe, M. C., Smith, J. C., Van Cleve, J. E., et al., 2012, arXiv:1203.1383

[8] Vanderburg, A., Johnson, J. A. 2014, PASP,126, 948

[9] Vanderburg, A. 2014, arXiv:1412.1827v1

[10] Van Cleve1, J. E., Howell, S. B., Smith J. C., 2015, arXiv: $1512.06162 \mathrm{v} 3$ 